\title{
Ein Blumenstrauss, der inspiriert, aber nicht alle Wünsche erfüllen kann
}

\section{Rouven Porz,}

Christina aus der $\mathrm{Au}$

Redaktion Ethik
Autonomie und Selbstbestimmung, Verantwortung und Fachwissen, Ethikdialog und Ethiktransfer - dies sind wegweisende Konzepte des Zürcher Instituts «Dialog Ethik». Das Institut Dialog Ethik spielt aus medizinethischer Sicht eine Pionierrolle im Schweizer Gesundheitssystem. Es leitet, entwickelt und begleitet seit Jahren Ethik-Foren an Kliniken, Spitälern und anderen Institutionen und es bietet ein reichhaltiges Fortbildungsprogramm zur Ethik im Gesundheitssystem an. Im Jahre 2009 hat Dialog Ethik nun ein fünfbändiges Ethik-Epos auf den Markt gebracht, das sogenannte «Handbuch Ethik im Gesundheitswesen». Die Titel der einzelnen Bücher widerspiegeln die Grundkonzepte von Dialog Ethik: Der selbstbestimmte Patient (Band 1); Ethikwissen für Fachpersonen (Band 2); Ethiktransfer in Organisationen (Band 3), Verantwortung im politischen Diskurs (Band 4) und Ethikdialog in der Wissenschaft (Band 5).

\section{Verknüpfung unterschiedlicher Zielsetzungen} Welch eine Bandbreite, welch ein Anspruch! Man kann die fünf Bände zusammen erwerben, sie erreichen die Leserin/den Leser in einer ansehnlichen Kartonbox, welche die 5 Bände beherbergt, ein eingängiges Layout, die einzelnen Bände farblich prägnant aufeinander abgestimmt, imposant. Es drängt sich allerdings die Frage des Anspruches auf. Welches Ziel verfolgen diese fünf Bücher? Ein Blick auf die erste Seite liefert eine erste Antwort. Auf jeder ersten Seite der Bücher findet sich derselbe Überblick: Band 1 will ein Ratgeber sein (für Menschen als Patienten), Band 2 versteht sich als Lehrbuch (für Berufsgruppen des Gesundheitssystems), Band 3 will Führungskräfte im Gesundheitssystem ansprechen, Band 4 wird als Ideengeber für den politischen Diskurs ausgewiesen, Band 5 setzt auf die Verbindung bzw. Einbettung in den aktuellen wissenschaftlichen Diskurs zur Medizin- bzw. Bioethik. Ein Blick ins Vorwort (das ebenfalls in allen 5 Bänden dasselbe ist) zeigt auf, wie diese unterschiedlichen Zielsetzungen miteinander verknüpft sind:

«(Das Handbuch) soll den Beteiligten helfen, mit den Fragen und Problemen ihrer jeweiligen Lebenswelt, soweit sie das Gesundheitssystem betreffen, umzugehen. Das Handbuch liefert damit nicht nur eine Gesamtschau ethischer Fragestellungen im Gesundheitssystem, sondern soll praxisnah und zielgruppengerecht Hilfestellung bieten, wie solche ethische Fra- gen bewusst, reflektiert und strukturenwirksam angegangen werden können.» (Vorwort, Seite 7).

Man versuche, sich diesen Anspruch noch einmal in aller Ruhe vor Augen zu führen: eine «Gesamtschau ethischer Fragestellungen», «zielgruppengerecht», und dann sollen alle diese Fragen auch noch «praxisnah» «angegangen» werden. Dann wären ja nach der Lektüre alle Probleme eigentlich geklärt! Dann wissen wir ja gleich, wie man das Gesundheitssystem in allen Ebenen ethischer gestaltet, sogar zielgruppengerecht, und wir können die Strukturen direkt implementieren! Okay, wir sind gespannt.

\section{Subjektiver Blick auf den «selbstbestimmten Patienten»}

Der erste Band für den selbstbestimmten Patienten ist herausgegeben von Ruth Baumann-Hölzle und Diana Meier-Allmendinger. Stellen wir uns also vor, wir seien Patienten. Das ist nicht so schwer, sind wir doch alle irgendwo bzw. irgendwann einmal Patienten oder auch Patientinnen. Wie in einem Blumenstrauss bieten uns die von den unterschiedlichsten Autoren geschriebenen Texte des ersten Bandes unterschiedliche Einblicke in die medizinethischen Grundkonzepte: zum Respekt vor der Patientenautonomie, zur Patientenverfügung, zum Kind, zum Jugendlichen, zur Frau und zum Mann als Patient.

Die Artikel bieten Perspektivenwechsel, Einblicke, Definitionen, immer sehr stark an die Person des jeweiligen Autors des jeweiligen Textes gebunden. Die Perspektiven bleiben somit subjektiv, aber vielleicht soll das ja gerade gezeigt werden. Ein kurzer Blick auf behinderte Patienten, alte Patienten, chronisch Kranke, Sterbende. Es fehlt allerdings ganz deutlich ein Hinweis auf Differenzierung, behindert ist nicht gleich behindert, alt ist nicht gleich alt, und sterbend schon gar nicht sterbend. Aber wir lesen diesen ersten Band ja als Patient, nicht als der oder die Wissenschaftler(in), der diese Rezension schreibt, vielleicht wäre uns als Patienten die mangelnde Ausdifferenzierung nicht aufgefallen.

Der erste Band endet mit einem «Autonomie-Ratgeber», der hier einer besonderen Betrachtung bedarf. Ein vermeintlich komplexes klinisches Fallbeispiel eines 16-jährigen Jungen wird erzählt, dann schrittweise aufgezeigt, wie der Junge seine Autonomie wahren kann, will oder soll. Was wir aber tatsächlich fin- 
den, sind ausführliche Exkurse zu den rechtlichen Zusammenhängen. Diese Darstellung karikiert die Selbstbestimmung des Patienten als eine juristische Angelegenheit - was mit moralisch-ethischer Autonomie leider nicht mehr viel zu tun hat!

\section{Durchdachte Beiträge - \\ fehlender roter Faden}

Der zweite Band, Ethikwissen für Fachpersonen, versteht sich als Lehrbuch und wurde von Christof Arn und Tatjana Weidmann-Hügle herausgegeben. In einem ersten Teil sollen die Werte von Fachpersonen behandelt werden, dann wird Ethik als Kompetenz und Werkzeug vorgestellt, abschliessend werden diverse ethische Fragestellungen in Medizin und Pflege aufgezeigt.

Wieder ein Blumenstrauss an Problemen und Sichtweisen, gut, um einen Überblick zu gewinnen, aber passt dieser Überblick zum Anspruch eines «Lehrbuches»? Die einzelnen Beiträge der einzelnen Autoren sind oftmals klar durchdacht, aber der rote Faden zum Gesamtbuch ist nicht immer erkennbar. Dieser Mangel liegt nicht in den Texten selbst, sondern am Anspruch der Herausgeber, dass hier «ethisches Fachund Methodenwissen» vermittelt werden soll (Seite 2, Überblick).
Dieser Anspruch passt nicht zu den meisten Texten, die ihrer jeweiligen Perspektive verhaftet bleiben. Es wäre realistischer gewesen, mit diesem Band einen Einblick in die Komplexität medizinethischer Probleme geben zu wollen. Zusammen mit einer reflektierten redaktionellen Betreuung hätte dies dem Anspruch eines Lehrbuches dann durchaus wieder entsprechen können.

\section{Transparenz und Offenheit als Vorzüge}

Ähnliche strukturelle Probleme ziehen sich durch die Bände 3, 4 und 5. Der hochgesteckte Anspruch kann von den zusammengestellten Einzeltexten nicht zusammenhängend eingelöst werden. Natürlich heisst dies nicht, dass es den Einzeltexten an Qualität fehlt. Ganz im Gegenteil! Einige Beispiele sollen unsere positiven Eindrücke hier exemplarisch illustrieren: Wenn Carlo Foppa z.B. im dritten Band (Ethiktransfer in Organisationen, hrsg. von Ruth Baumann-Hölzle und Christof Arn) von seinen eigenen Erfahrungen als klinischer Ethiker berichtet, dann wird die Komplexität der Implementierung von Ethik deutlich. Wenn Markus Zimmermann im vierten Band (hrsg. von Markus Christen und Max Baumann) die Knappheit in der Gesundheitsversorgung beschreibt, dann spürt man regelrecht, dass die Komplexität der Rationierungs-

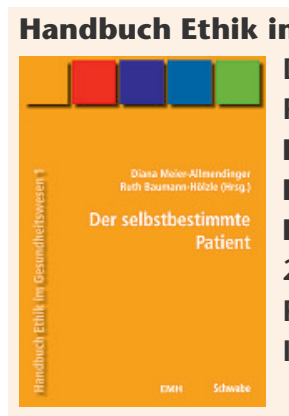

\section{im Gesundheitswesen \\ Diana Meier-Allmendinger, \\ Ruth Baumann-Hölzle (Hrsg.) \\ Band 1: \\ Der selbstbestimmte \\ Patient \\ 2009. 276 Seiten. Broschiert \\ Fr. 55.- / $€ 38.50$ \\ ISBN EMH 978-3-03754-036-7}

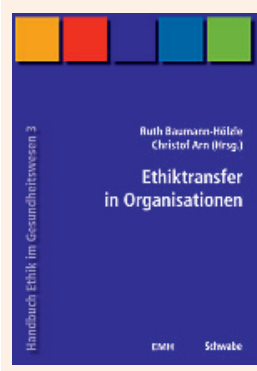

Ruth Baumann-Hölzle,

Christof Arn (Hrsg.)

Band 3: Ethiktransfer in

Organisationen

2009. 284 Seiten. Broschiert.

Fr. 55. - $€ 38.50$

ISBN EMH 978-3-03754-038-1

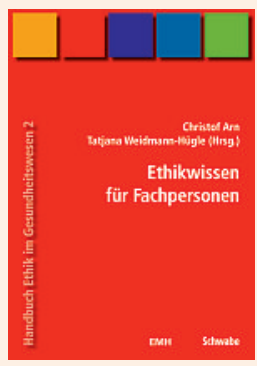

Christof Arn,

Tatjana Weidmann-Hügle

(Hrsg.)

Band 2: Ethikwissen

für Fachpersonen

2009. 308 Seiten. Broschiert.

Fr. 55.- / $€ 38.50$

ISBN EMH 978-3-03754-037-4

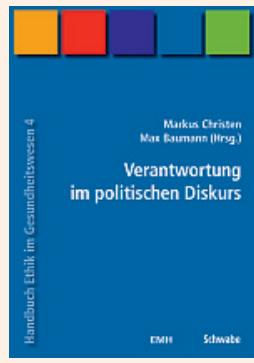

Markus Christen,

Max Baumann (Hrsg.)

Band 4: Verantwortung

im politischen Diskurs

2009. 240 Seiten. Broschiert.

Fr. 55.- / € 38.50

ISBN EMH 978-3-03754-039-8

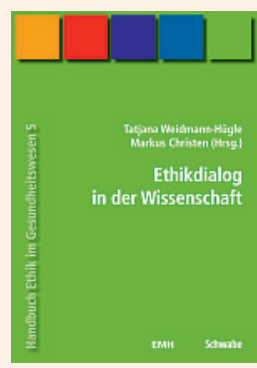

Tatjana Weidmann-Hügle,

Markus Christen (Hrsg.)

Band 5: Ethikdialog

in der Wissenschaft

2009. 244 Seiten. Broschiert.

Fr. 55.- / $€ 38.50$

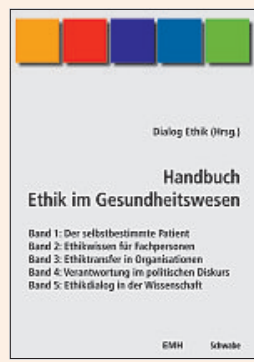

Dialog Ethik (Hrsg.)

Schuber Band 1-5:

Handbuch Ethik

im Gesundheitswesen

2009. 5 Bände.

Fr. 248.- / $€ 173.60$

(statt Fr. 275.- / € 192.50)

ISBN EMH 978-3-03754-041-1 
debatte nicht mit einfachen Modellen angegangen werden kann. Und wenn Urs Thurnherr, Giovanni Maio und Dietmar Mieth im fünften Band (hrsg. von Tatjana Weidmann-Hügle und Markus Christen) kritische Erwiderungen an die Ausführungen zur Integrativen Verantwortungsethik von R. Baumann-Hölzle und C. Arn adressieren, dann befinden wir uns im Kern der akademischen Debatte um unterschiedliche Auffassungen der Aufgabe(n) von Ethik.

Gerade der letzte Punkt verweist auf einen - aus unserer Sicht - besonders lobenswerten Aspekt der fünf Bände: Dialog Ethik stellt sich in seinen Überlegungen hier ganz offen und transparent zur Schau. Die Mitglieder des Dialog-Ethik-Teams zeigen, wie sie denken, was sie machen und welchen moralischen Anspruch sie haben. Dies wird insbesondere in den Bänden 3 und 5 deutlich, wenn Dialog Ethik andere Autoren aktiv einlädt, kritische Erwiderungen und Kommentare zu ihren eigenen Konzepten und zu ihrem eigenen Entscheidungsfindungsmodell zu schreiben. Diese offene Transparenz in der Zur-Schau-Stellung der eigenen Thesen und die gleichzeitige Aufforderung, diese eigenen Thesen konstruktiv-kritisch zu hinterfragen, fehlt oft in der medizinethischen Diskussion. den fünf Bänden um «Sammelbände» handelt, so hat es sich die Herausgeberschaft von Dialog Ethik nicht nehmen lassen, ein Gesamtlayout über alle Beiträge zu legen. Dieses Gesamtlayout wird als «redaktioneller Beitrag» bezeichnet (Seite 6 jedes Bandes) und besteht darin, dass alle Beiträge mit Definitionen, Exkursen, ja gar mit zusammenfassenden Sätzen belegt sind. Es lässt sich nicht leugnen, dass insbesondere die Zusammenfassungen am Rand der Texte ganz deutliche Interpretationen von Dialog Ethik zum Geschriebenen der Autoren und Autorinnen sind.

Diese Vorgehensweise ist in Sammelbänden sehr fragwürdig, weil dem Lesenden nicht immer klar ist, welche Aussage jetzt vom Einzelautor und welche von Dialog Ethik stammt. Leider findet sich eine vergleichbare methodische Unschärfe auch in der Präsentation der meisten Fallbeispiele. Die Fallbeispiele transportieren generell eine moralische Botschaft und sind so zu den Texten ausgewählt, dass dem Lesenden kaum Raum für eigene Interpretationen bleibt. Der Fall dient zu oft der Untermauerung der Argumentation von Dialog Ethik und zu wenig der Anregung zum freien Denken des Lesenden.

\section{Das fünfbändige Epos liefert eine Fülle an Perspektiven und Themen, sinnvoll und hilfreich für jeden, der sich mit grundlegenden ethischen Fragen im Gesundheitssystem befassen will. Der eigene Anspruch der Herausgeberschaft ist aber zu hoch}

Die Kehrseite der Medaille ist leider ein weiterer Kritikpunkt am Gesamtwerk: Dieses scheinbar kritische Zur-Diskussion-Stellen der eigenen Arbeit könnte auch als Eigenwerbung gedeutet werden, als strategischer Schritt, um den Nutzen des eigenen Instituts im Schweizer Kontext voranzutreiben. Die Grenze dieser strategischen Eigenwerbung überschreitet R. BaumannHölzle aus unserer Sicht deutlich im dritten Band, wenn sie Sinn und Nutzen des von ihr selbst implementierten Ethik-Forums am Kinderspital Zürich in den Fokus rückt, in dem sie die dort verantwortlichen Mediziner, Pflegedienstleitung und ärztlichen Direktor zur eigenen Arbeit von Dialog Ethik befragt. Dieses Interview wäre besser von anderer Seite geführt worden. Eine solche Vermischung von eigenen Interessen, insbesondere in einem Band, der sich an Führungskräfte in Spitälern wendet, scheint unangebracht, gar hinderlich und unnötig, um die unzweifelhaft sinnvolle Arbeit von Dialog Ethik in ein konfliktfreies Licht zu rücken.

Dieser Anmerkung lässt sich eine weitere anfügen, die alle fünf Bände betrifft. Obwohl alle fünf Bände jeweils aus Einzelbeiträgen von Einzelautoren und -autorinnen bestehen, obwohl es sich also sozusagen bei

\section{Noch sind nicht alle Probleme gelöst}

Summa summarum: Das fünfbändige Epos liefert eine Fülle an Perspektiven und Themen, ein von Dialog Ethik gebundener Blumenstrauss, sinnvoll und hilfreich für jeden, der sich mit grundlegenden ethischen Fragen im Gesundheitssystem befassen will. Der eigene Anspruch der Herausgeberschaft ist aber zu hoch. Konstruktive Kritik wird von Dialog Ethik in seinen Werken explizit eingefordert. Deshalb nimmt die vorliegende Rezension diese Aufforderung ernst: Wir haben uns in einem Zweierteam bemüht, positive Seiten zu betonen, aber wahrgenommene Mängel, insbesondere methodischer Art, auch nicht zu vertuschen.

Dabei muss natürlich die subjektive Sichtweise der Rezensierenden und deren eigene wissenschaftliche Ausrichtung bedacht werden, vor allem deren Vorliebe für feministische, narrative und kontextuelle Ansätze der Biomedizinischen Ethik, die in den fünf Bänden in der Dominanz der Betonung auf Entscheidungsfindung und individuell-rationaler Patientenautonomie leider etwas untergehen. Der Blumenstrauss war inspirierend und anschaulich, aber die Suche geht weiter, noch sind nicht alle Probleme gelöst. 